\title{
$\operatorname{coo}-3352-6$
}

ANNUAL PROGRESS REPORT (NSMRG-6)

to

Division of Research

United States Atomic Energy Commission

THERMAL NEUTRON SCATTERING STUDIES OF MOLECULAR

DYNAMICS AND CRITICAL PHENOMENA IN FLUIDS

Contract AT $(11-1) 3352$

Period Covered: Dec.1973-Nov.1974

\begin{abstract}
Neutron Spectroscopy and Materials Research Group Massachusetts Institute of Technology
\end{abstract}

Principal Investigator

Associate Investigator

DSR Staff

Research Assistant

Research Assistant
Professor S. H. Chen

Professor S. Yip

Dr. H. C. Teh

T. A. Postol

W. Veldkamp

$\therefore \cdots$ s. !n...

\section{NoTice $\cdots$}

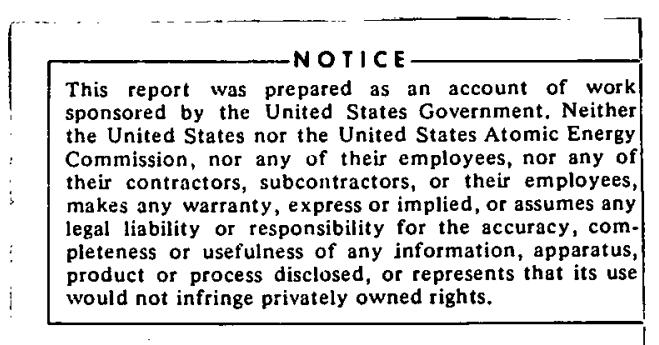

PORTIONS OF THIS REPORT ARE ILLEEIBLE. II

has been reproduced frem the best availatide copy to permit the broadest possible ayalf

ability. 


\section{DISCLAIMER}

This report was prepared as an account of work sponsored by an agency of the United States Government. Neither the United States Government nor any agency Thereof, nor any of their employees, makes any warranty, express or implied, or assumes any legal liability or responsibility for the accuracy, completeness, or usefulness of any information, apparatus, product, or process disclosed, or represents that its use would not infringe privately owned rights. Reference herein to any specific commercial product, process, or service by trade name, trademark, manufacturer, or otherwise does not necessarily constitute or imply its endorsement, recommendation, or favoring by the United States Government or any agency thereof. The views and opinions of authors expressed herein do not necessarily state or reflect those of the United States Government or any agency thereof. 


\section{DISCLAIMER}

Portions of this document may be illegible in electronic image products. Images are produced from the best available original document. 


\section{Introduction}

Our activity during this period can be classified in three categories: coherent and incoherent neutron scattering in dense gases; development of a photon correlation spectrometer for the study of critical phenomena; and theoretical work in the kinetic theory of dense gases. Owing to the shutdown of the MIT nuclear reactor in May 1974, we were not able to perform much of the originally planned experimental work in liquids; however, we were able to make two significant measurements in dense gases. Coherent scattering from nitrogen gas at a range of pressures up to 512 atm was performed at Brookhaven National Laboratory. Assistance from Dr. Lawrence Passell of BNL in the measurement was greatly appreciated. Incoherent scattering from hydrogen gas at pressures up to $2000 \mathrm{~atm}$ was done at Argonne National Laboratory and again the assistance of Dr. Kurt Skold of ANL was invaluable.

Dr. H. C. Teh stayed with us for a period of eleven months, leaving us for the University of Singapore at the end of April 1974. He and T. A. Postol made most of the neutron measurements in gases. These results, which are being written up for publication, will also constitute the PhD dissertation of T. A. Postol, entitled "Experimental Test of Non-Classical Kinetic Theory in Dense Gases". .

S. H. Chen and W. Veldkamp developed a digital correlator in the form of a compact, integrated unit. It will be at the heart of a computerized photon correlation spectrometer now being completed at oux laboratory. 
S. Yip and T. A. Postol developed a digital computer program which is capable of calculating the dynamic structure factor of dense gases corresponding to conditions under which the neutron experiments were done. The theory underlying the calculations was formulated by S. Yip and his students.

We summarize the achievements of this period in the following list of articles and papers published or to be published.

1. Spectroscopy in Biology and Chemistry - Neutron, X-ray, Laser, edited by S. H. Chen and S. Yip, Academic Press (1974). In this book there are the following three articles written under the present contract:

a) S. H. Chen, "Introduction to Neutron, X-ray and Laser Spectroscopy"

b) S. Yip;" "Quasielastic Scattering in Neutron and Laser spectroscopy"

c) S. H. Chen, "Photon Correlation Spectroscopy in Biology"

2. O. L. Deutsch and S. Yip, "Van Hove Self Correlation Functions in a Maxwell Gas", Phys. Fluids 17, 252 (1974).

3. S. H. Chen, W. B. Veldkamp and C. C. Lai, "A Simple Digital Clipped Correlator for Photon Correlation Spectroscopy" (to be published).

4. S. H. Chen, T. A. Postol and K. Skold, "Observation of Collective Enhancement of Diffusion Coefficient of Hydrogen Gas at High Densities" (to be published).

5. S. H. Chen, T. A. Postol, H. C. Teh and S. Yip, "Observation of Brillouin Scattering of Neutrons from Nitrogen Gas at High Densities" (to be published). 
II. Research Performed

A. Coherent Scattering from Dense Nitrogen Gas

The central problem in current research in neutron inelastic scattering in simple fluids is the study of dynamic structure factor $S(Q, \omega)$, the frequency-dependent generalization of $S(Q)$. A measurement of $S(Q, \omega)$ gives directly information about the temporal and spatial dependence of density fluctuations in the fluid. Because neutron scattering involves frequencies and wavelengths which are comparable to inter-particle collision frequencies and separations, neutron data are particularly useful for studying transport and fluctuation processes at the molecular level. Computer molecular dynamics calculations also provide information at these frequencies and wavelengths, and by combining the two kinds of results one has the most detailed and relevant data available for investigating the dynamical and structural behavior of simple fluids.

Recently extensive neutron measurements of $\$(R, \omega)$ and the single-particle form factor $s_{s}(Q, \omega)$ have been made for argon near the triple point ${ }^{[1]}$. Data analysis showed that for the range of $Q$ values measured, namely between 1.0 and $4.4 \AA^{-1}$, the results could be quite well explained by generalized hydrodynamic calculations [2], [3]. While the method of generalized hydrdynamics offers a convenient calculationalprocedure it is not suitable for a detailed understanding of the molecular processes which govern the behavior of $S(Q, w)$. One needs a theoretical framework in which dynamical processes can be discussed at the molecular level. Such an approach is provided by by kinetic theory. The kinetic equation conventionally used to discuss transport properties of dense gases and liquids is the Enskog equation [4]. 
The Enskog equation is an intuitive extension of the Boltzmann equation where one now takes into account the finite size of the colliding molecules and the effects of collisional transfer. When the Enskog equation is modified to satisfy sum-rule properties [5] it is a reasonable starting point for discussing transport coefficients as well as short-wavelength fluctuations.

The validity of the modified Enskog equation to describe density fluctuations at frequencies and wavelengths appropriate to neutron scattering is therefore of considerable interest. In a preliminary study we have carried out coherent neutron scattering measurements on $\mathrm{N}_{2}$ gas pressurized up to 512 atm [6] . In Fig. 1 the observed scattering intensity is plotted against dimensionless frequency for two gas densities and different experimental resolutions. At higher density and better resolution (upper curve) the Brillouin doublet structure familiar in light scattering studies of fluids is clearly visible. Although the Brillouin structure has been observed in neutron measurements on liquid rubidium[7], parahydrogen $[8]$, neon $[9]$ and lead ${ }^{[10]}$, ours is the first measurement in the gas phase. In the case of argon such structure has not been seen in either the Iiquid ${ }^{[1]}$ or the gas ${ }^{[11]}$ data. It is important to note that for coherent scattering at small $Q$ there are definite advantages in making the measurements in the gas phase. In going from the liquid to the vapor phase the structure factor at small $Q$ can increase by as much as a factor of 10. Furthermore, it is much more useful to have experimentaj $S(Q, \omega)$ over a wide range of densities in testing current theories. 


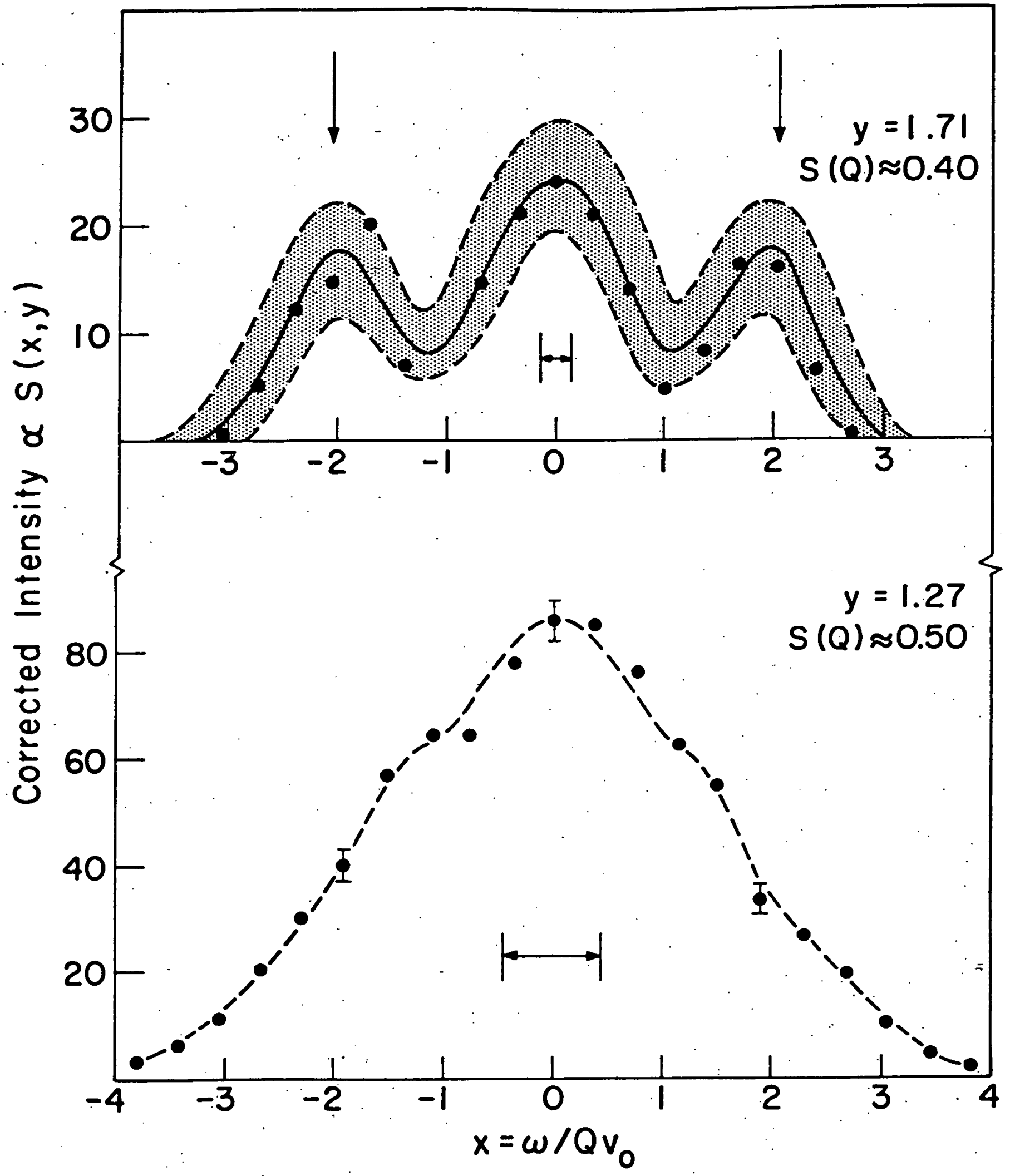

Figure 1 


\section{References}

1. K. Skold, J. M. Rowe, G. Ostrowski and P. D. Randolph, Phys. Rev. 6A, 1107 (1972).

2. J. M. Rowe and $K$. Skold, in Neutron Inelastic Scattering,

IAEA (1972) p. 413 and the references therein.

3. See review article by Egelstaff in Ann. Rev. Phys. Chem. 24, 159 (1973).

4. See, for example, H. J. M. Hanley, R. D. McCarty and E. G. D. Cohen, Physica 60,322 (1972).

5. J. Sykes, J. Stat. Phys. 8,279 (1973); H. H. V. Konijnendijk and J. M. J. van Leeuwen, Physica 64, 342 (1973).

6. H. C. Teh, T. A. Postol, S. H. Chen and S. Yip, Bull. Am. Phys. Soc. series II, 19 paper KKI7 (1974), and to be published.

7. J. R. D. Copley and J. M. Rowe, Phys. Rev. Lett. 32, 49 (1974);

J. R. D. Copley and J. M. Rowe, Phys. Rev. A9, 16 $\overline{56}$ (1974).

8. K. Carneiro, M. Nielsen, and J. P. McTague, Phys. Rev. Lett. 30, 481 (1973).

9. H. G. Bell, A. Kollmar, B. Alefeld, and T. Springer, Phys. Letters 45A, 479 (1973).

10. B. Dorner, T. Plesser, and H. Stiller, Discuss. Faraday Soc. 43, 160 (1967).

11. J. J. van Loef, in Neutron Inelastic Scattering 1972, (International Atomic Energy Agency, Vienna, 1972), p. 429. 
B. Incoherent Scattering from Dense Hydrogen Gas

We have in the previous year measured the incoherent scattering law $S_{S}(Q, \omega)$ at $85^{\circ} \mathrm{K}$ for densities up to $1.09 \times 10^{22}$ particles $/ \mathrm{cm}^{3}$. The data as analyzed in [1] showed that the Enskog kinetic theory is adequate to explain both the self diffusion coefficient D and the line shape of $S_{S}(Q, \omega)$. However, the validity of the Enskog equation as a quantitative description of transport properties of dense gases has recently been seriously challenged by computer studies of the long-time behavior of time correlation functions [2]. It was found that collective effects which appear to involve particle clusters cause the correlation functions to decay like a power law rather than an exponential as implied by the Enskog equation. The Enskog description which does not consider this type of collective effect therefore will not give the correct transport coefficient values at sufficiently high densities. We therefore undertook a measurement at room temperature with pressures of 1000,1500 and 2000 atms, corresponding to densities $1.49 \times 10^{22}, 1.87 \times 10^{22}$ and $2.16 \times 10^{22}$ particles $/ \mathrm{cm}^{3}$. Considering the critical density of hydrogen $n_{c}=0.934 \times 10^{22}$ the densities we have reached are those of the liquid densities.

The analysis of data is complicated because we wish to obtain absolute values of $S_{S}(Q, \omega)$ and therefore the multiple scattering correction is very important. We also extracted the resolution effects of the time-of-flight spectrometer used. The preliminary data analysis thus shows that we have at low Q's (small angles) very nice Lorentzian lines for $s_{s}(Q, \omega)$ and therefore could extract unambiguously the self diffusion coefficient. The values obtained 
are $2.05 \times 10^{-3}, 1.46 \times 10^{-3}$ and $1.15 \times 10^{-3} \mathrm{~cm}^{2} / \mathrm{sec}$. By also computing $D_{E}$ (diffusion coefficient according to the Enskog theory) we have obtained the ratio $D / D_{E}$ at the three densities to be 1.20 , 1.30 and 1.32 . We have thus unambiguously verified the effect predicted by the computer simulation work, namely the long-time tail in the velocity autocorrelation function of the test particle and the associated enhancement of the diffusion coefficient. We are currently analyzing the data at larger $Q$ to see whether we can also observe the deviation from the Enskog theory in the line shape of $S_{S}(Q, \omega)$

\section{References}

1. S. H. Chen, Y. Lefevre and S. Yip, Phys. Rev. 8A, 3163 (1973).

2. See the review by B. J. Alder, in Transport Phenomena - 1973, AIP Conference Proceedings No. 11, J. Kestin, ed. (AIP, New York, 1973), ए. 1 . 
C. Development of a Photon Correlation spectrometer

During the last several years we have been designing, developing and improving a photon correlation spectrometer. We feel we have reached the final stage of the development and therefore are ready to finalize the product. Fig. 2 shows the schematic layout of the spectrometer we have now at our laboratory. The most important signal processing unit is the 128 channel clipped digital correlator which we designed from scratch. The next two figures show the details of the 16-channel integrated eircuit card that we now produce. We are rather proud of this part of the instrumentation and are publishing an article about it so that other researchers can share our experiences.

D. Theoretical Work in Kinetic Theory of Dense Gas.

The so-called generalized Enskog equation applicable to dynamics of dense gases was developed by Mazenko[1], sykes [2] and Konijnendijk and $\operatorname{Van}$ Leeuwen [3]. However, the solution of this equation using the method of kinetic model was carried out by Castresana and Yip ${ }^{[4]}$. The computer program which computes the $S_{S}(Q, \omega)$ and $S(Q, W)$ for dense gases with an effective hard core interaction was recently completed by Yip and Postol. We are thus in a position to analyze all our data with this program.

\section{References}

1. G. F. Mazenko, Phys. Rev. A9, 360 (1974).

2. J. Sykes, J. Stat. Phys. 8,279 (1973).

3. H. H. V. Konijnendijk and J. M. J. Van Leeuwen, Physica 64,342 (1973).

4. J. Castresana, "Generalized Boltzmann Equation Description of Density Fluctuations in Fluid Mixtures", PhD thesis, MIT (1974). 


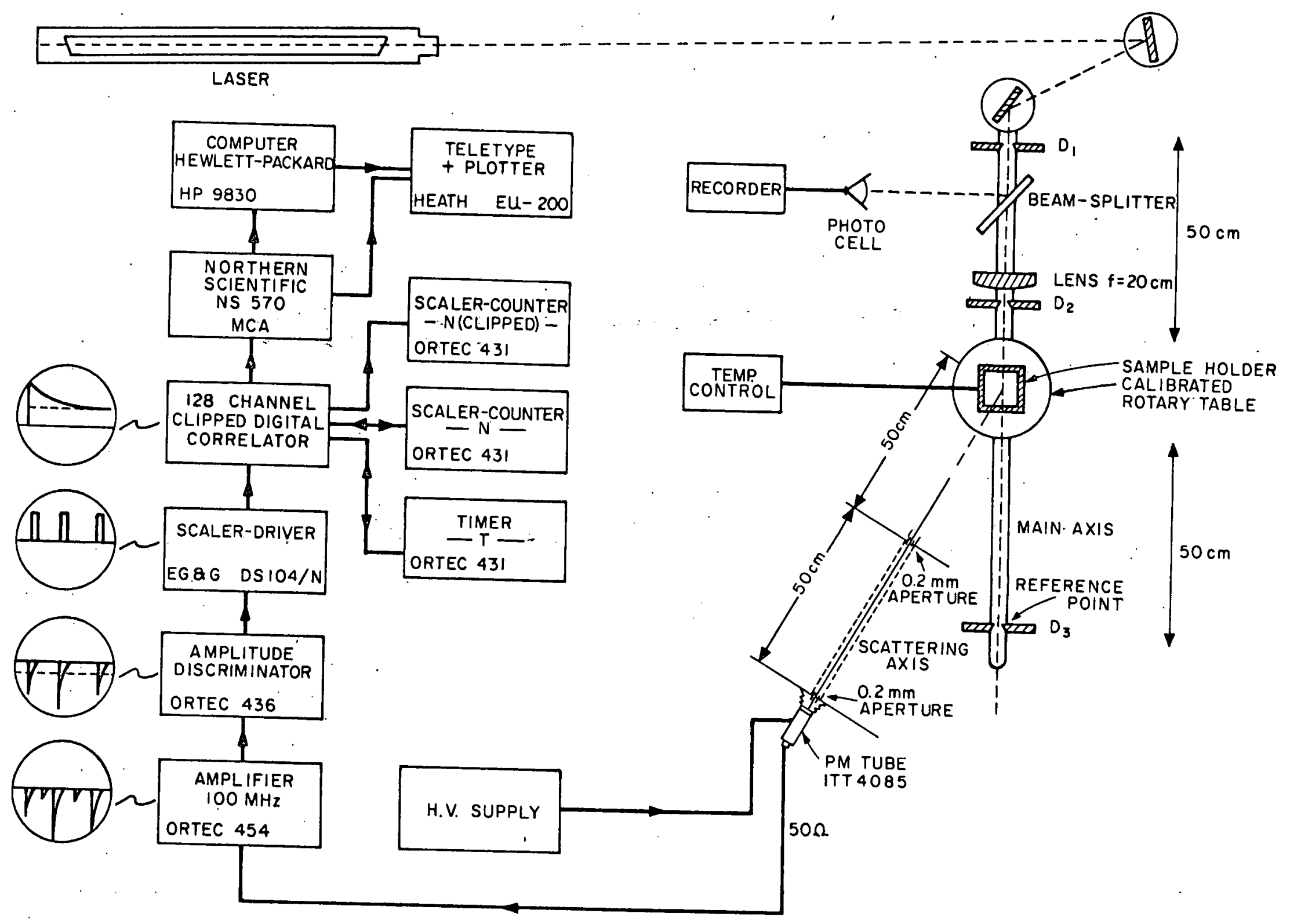

A PHOTON CORRELATION SPECTROMETER 


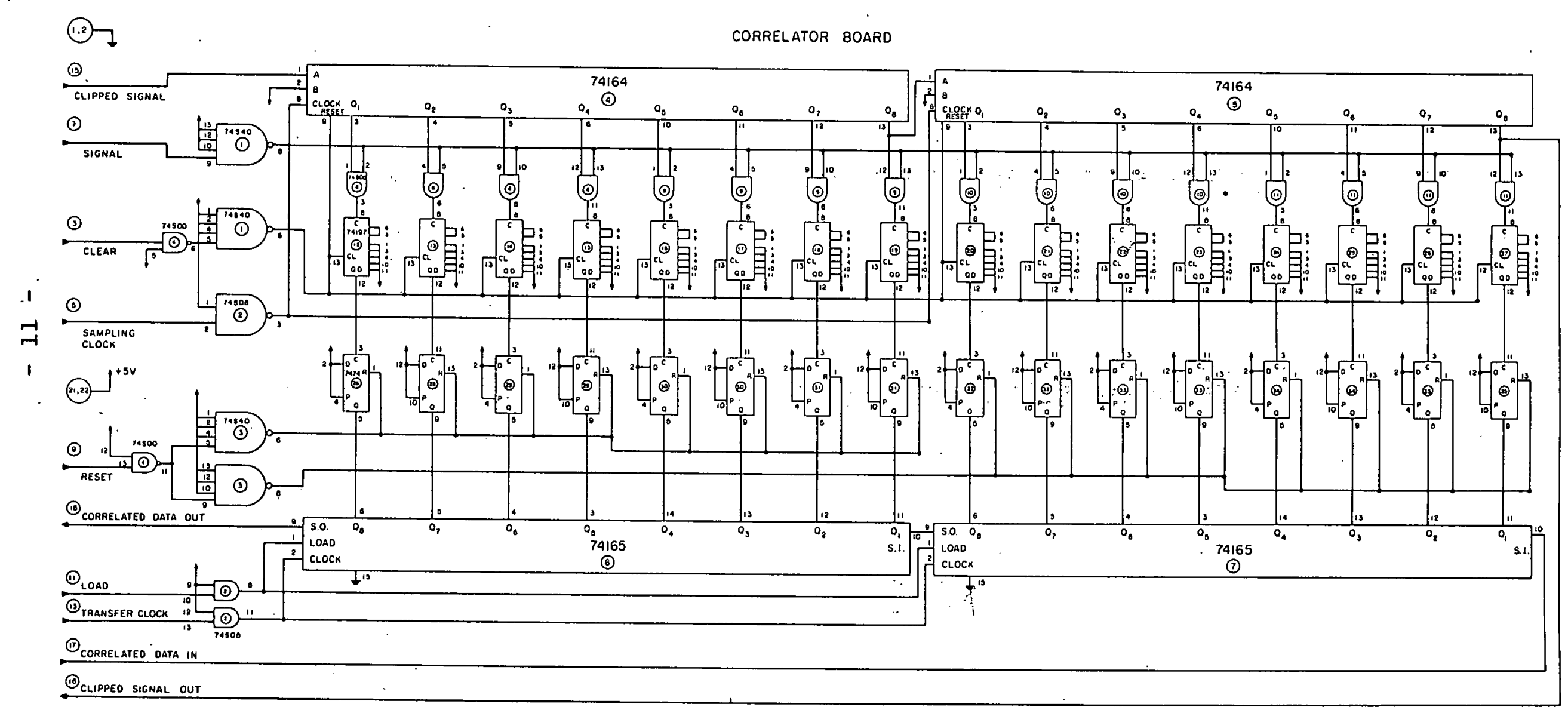




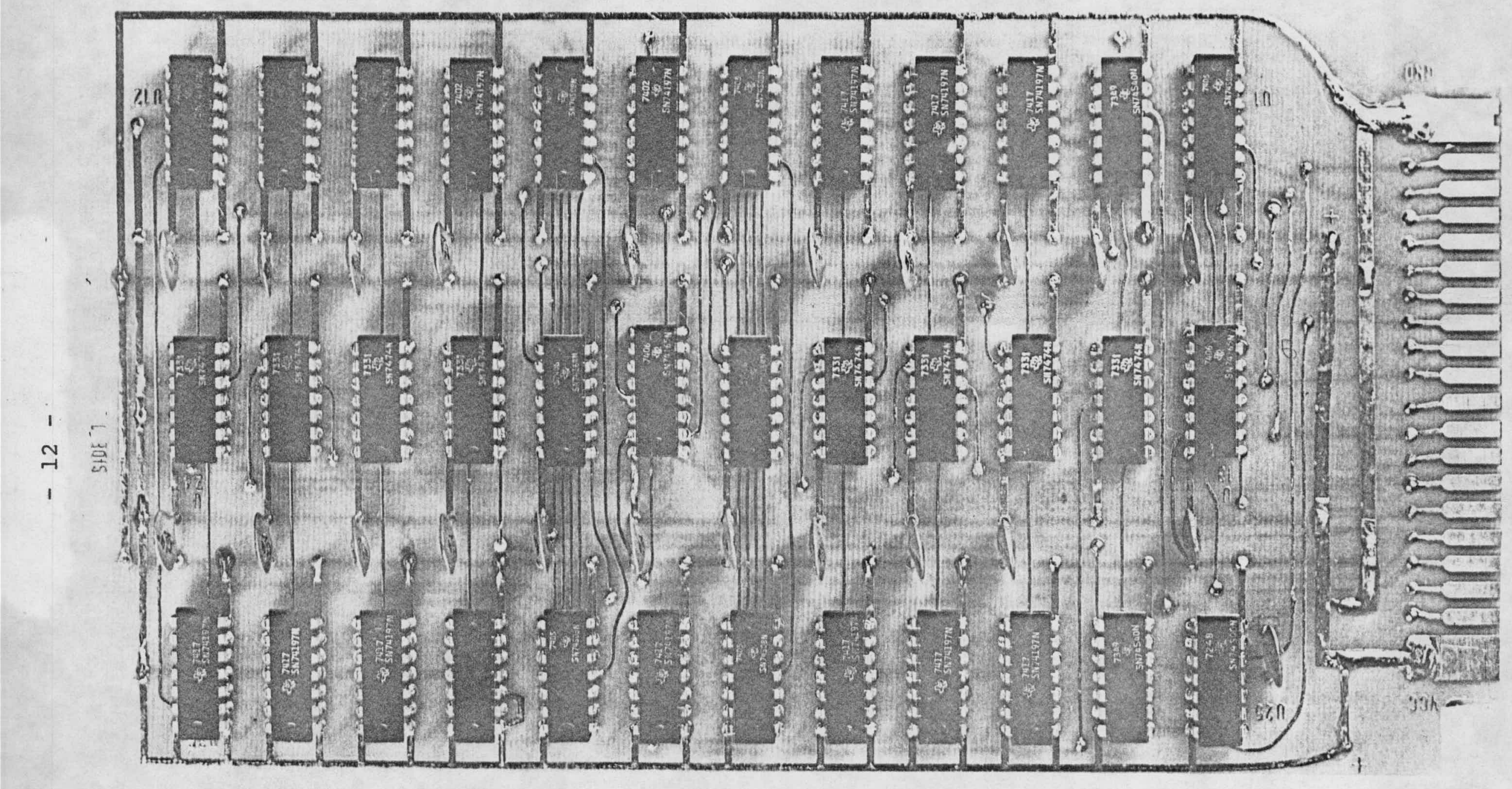

